\title{
Magnetic properties of single rare-earth atoms on graphene/Ir(111)
}

\author{
Romana Baltic, ${ }^{1}$ Fabio Donati, ${ }^{2,1,3}$ Aparajita Singha, ${ }^{1}$ Christian Wäckerlin, ${ }^{1,4}$ Jan Dreiser, ${ }^{1,5}$ \\ Bernard Delley, ${ }^{6}$ Marina Pivetta, ${ }^{1}$ Stefano Rusponi, ${ }^{1}$ and Harald Brune ${ }^{1, *}$ \\ ${ }^{1}$ Institute of Physics, Ecole Polytechnique Fédérale de Lausanne, CH-1015 Lausanne, Switzerland \\ ${ }^{2}$ Center for Quantum Nanoscience, Institute for Basic Science (IBS), Seoul 03760, Republic of Korea \\ ${ }^{3}$ Department of Physics, Ewha Womans University, Seoul 03760, Republic of Korea \\ ${ }^{4}$ Empa, Swiss Federal Laboratories for Materials Science and Technology, 8600 Dübendorf, Switzerland \\ ${ }^{5}$ Swiss Light Source, Paul Scherrer Institut, CH-5232 Villigen, Switzerland \\ ${ }^{6}$ Paul Scherrer Institut WHGA 123, CH-5232 Villigen, Switzerland
}

(Received 8 December 2017; published 13 July 2018)

\begin{abstract}
We employed x-ray absorption spectroscopy and x-ray magnetic circular dichroism to study the magnetic properties of single rare-earth (RE) atoms (Nd, Tb, Dy, Ho, and Er) adsorbed on the graphene/Ir(111) surface. The interaction of RE atoms with graphene results for $\mathrm{Tb}$ in a trivalent state with $4 f^{n-1}$ occupancy, and in a divalent state with $4 f^{n}$ occupancy for all other studied RE atoms ( $n$ corresponds to the $4 f$ occupancy of free atoms). Among the studied RE on graphene/Ir(111), Dy is the only one that shows magnetic hysteresis and remanence at $2.5 \mathrm{~K}$. By comparing measured spectra and magnetization curves with multiplet calculations, we determine the energy diagram of the magnetic states and show for each element the magnetization reversal process that determines the timescale of its magnetic bistability.
\end{abstract}

DOI: 10.1103/PhysRevB.98.024412

\section{INTRODUCTION}

The demand for storing and processing information at ever-smaller scales has driven research toward isolated surface adsorbed atoms as bit units. Besides their potential in future storage technology, such atoms represent valuable model systems for investigating fundamental magnetic phenomena and conditions required for stable magnetic quantum states [1-11]. Rare-earth (RE) atoms possess an intrinsic large spinorbit interaction and their magnetic states are well decoupled from the environment. These properties have been exploited to design single ion molecular magnets with magnetic bistability on a long timescale [12-16]. Magnetic remanence and long magnetic lifetimes have also been achieved in single RE adatoms. The two first, and so far only, systems of single atom magnets are $\mathrm{Ho}$ atoms on $\mathrm{MgO} / \mathrm{Ag}(100)[17,18]$ and $\mathrm{Dy}$ atoms on graphene/Ir(111) [19].

As demonstrated in Ref. [19], a single layer of graphene not only allows us to self-assemble RE atoms in ordered arrays, but also decouples them from electrons and phonons of the underlying $\operatorname{Ir}(111)$ substrate and thereby stabilizes their magnetic moment at $2.5 \mathrm{~K}$. The origin of these remarkable magnetic properties is twofold: First, the high $C_{6 v}$ crystal field (CF) symmetry provided by the adsorption on graphene hollow site results in a ground-state doublet that is protected from direct quantum tunneling of the magnetization (QTM); second, graphene high stiffness hinders the exchange of energy and momentum with phonons, while the energy gap around the Fermi energy $\left(E_{F}\right)$ and the weak interaction with $\operatorname{Ir}(111)$ limit the transmission of electrons from the substrate through the graphene layer.

*harald.brune@epfl.ch
A deeper understanding of the interaction that results in the remarkable magnetic stability of Dy atoms would enable tailoring the magnetic properties of RE atoms on graphene and other two-dimensional materials. So far, only very few theoretical predictions for the magnetic behavior of RE atoms adsorbed on a supporting surface have been reported [20-23]. Therefore, one way to derive a better understanding is to explore experimentally the behavior of several RE elements possessing different electronic configurations. Such surface-supported RE atoms represent model systems for classical and quantum magnetic storage, providing a benchmark for future theoretical developments. Here we use x-ray absorption spectroscopy (XAS), magnetic circular (XMCD), and linear dichroism (XMLD), and multiplet calculations to explore the magnetic stability and quantum level structure of $\mathrm{Nd}, \mathrm{Tb}, \mathrm{Ho}, \mathrm{Er}$, and compare them with similar measurements of Dy on graphene/Ir(111). We show that the $\mathrm{CF}$ generated by the graphene-RE interaction is element specific, as well as the corresponding splitting of the magnetic eigenstates. The latter allows us to identify the relaxation mechanisms of the magnetic states and to discuss the characteristic magnetic lifetime of the different RE atoms.

This paper is organized as follows. Section II gives the theoretical background. Section III describes details of sample preparation, experimental measurements, and multiplet calculations. In Sec. IV A, we present the magnetic properties of Dy atoms on graphene/Ir(111), which exhibit divalent configuration on this surface. We show a coverage dependent variation of their XAS, the influence of $\mathrm{x}$-ray photon flux on the hysteresis curves, as well as the effects of contamination on the XAS. In Sec. IV B, we present the magnetic properties of other divalent $\mathrm{RE}$ atoms (Nd, Ho and Er) on graphene/Ir(111), while Sec. IV C is dedicated to $\mathrm{Tb}$ atoms that are trivalent on this surface. We discuss the results in Sec. V, and conclude in Sec. VI. 


\section{THEORETICAL BACKGROUND}

The magnetic properties of RE atoms originate from their highly localized $4 f$ orbitals. The magnetic states of these atoms in the gas phase are well described with the total angular momentum $J$ and its projection $J_{z}$. Upon adsorption on a surface, $J$ remains a good quantum number due to the strong spin-orbit coupling and the weak interaction of the $4 f$ orbitals with the environment. The adsorption site of an atom on a surface determines the symmetry and the strength of the CF, which lifts the degeneracy of the $J_{z}$ states and mixes them. Density functional theory (DFT) calculations suggest the sixfold coordinated hollow site as adsorption site for RE atoms on freestanding graphene [24]. For Dy atoms on graphene/Ir(111), we determined this adsorption site experimentally [19]. A sixfold hollow site generates a $\mathrm{C}_{6 v} \mathrm{CF}$ to the adsorbed atoms. As described in Refs. [25,26], in $\mathrm{C}_{6 v}$ symmetry, the CF Hamiltonian can be expressed in terms of a reduced set of Stevens operators $\hat{O}_{m}^{n}$ :

$$
\hat{H}_{\mathrm{CF}}=B_{0}^{2} \hat{O}_{0}^{2}+B_{0}^{4} \hat{O}_{0}^{4}+B_{0}^{6} \hat{O}_{0}^{6}+B_{6}^{6} \hat{O}_{6}^{6},
$$

where $B_{m}^{n}$ are the parameters allowed by the symmetry of the $\mathrm{CF}$ environment. The first three parameters, $B_{0}^{2}, B_{0}^{4}$, and $B_{0}^{6}$, are the uniaxial parameters. They split different $J_{z}$ states and determine the total zero field splitting (TZFS), i.e., the energy difference between the lowest and the highest lying $J_{z}$ state. In this case, this value is equal to the height of the energy barrier required for thermally assisted magnetization reversal, $U_{\text {rev }}$, and, by analogy with classical systems, it is commonly related to the magnetic anisotropy energy. The last parameter, $B_{6}^{6}$, is the transverse CF parameter. It mixes $J_{z}$ states that differ by $\Delta J_{z}= \pm 6$, hence the resulting eigenstates of $\hat{H}_{\mathrm{CF}}$ are linear combinations of several $J_{z}$ states. This parameter can induce tunnel splitting between the levels of a doublet, thus introducing QTM into the system, and can open new relaxation channels via scattering with electrons and phonons of the substrate, both effects reducing the value of $U_{\text {rev }}$.

The necessary condition for magnetic stability is a ground state doublet that is not mixed in the CF symmetry, namely a doublet for which QTM does not occur. Increased stability of such a doublet, and hence of the atom's magnetization, can be achieved by suppressing scattering with electrons and phonons from the substrate that can give rise to additional relaxation pathways via excited states (thermally activated QTM). These two mechanisms are summarized in the following for both Kramers (half-integer $J$ ) and non-Kramers (integer $J$ ) atoms in $C_{6 v}$ symmetry:

(a) Quantum tunneling of magnetization. QTM is the direct consequence of the coupling between different $J_{z}$ states which are matching in energy [27]. The coupling between two $J_{z}$ states can be expressed in the following way:

$$
\left\langle J_{z}=n\left|\hat{O}_{6}^{6}\right| J_{z}=m\right\rangle \neq 0, \quad|n-m|=6,12 .
$$

In the case of integer $J$ systems, all doublets except $J_{z}=$ $\pm 3, \pm 6$ are protected against QTM. At $B=0 \mathrm{~T}$, these states form tunnel-split doublets with quenched $J_{z}$. If one of these doublets is the ground state, QTM occurs and there is no stable magnetization. In the case of half-integer $J$ systems, QTM is forbidden because $2 J_{z}$ is an odd number and thus, according to Eq. (2), the two levels of the doublet cannot be mixed.
In a deeper analysis, both Kramers and non-Kramers doublets can be classified into perfectly axial and nonaxial doublets [28]. This latter case includes the doublets allowing QTM identified by Eq. (2) and Kramers doublets that tunnel split in presence of additional perturbations leading to magnetization reversal, as described below.

(b) First-order transverse perturbations. Spin reversal can also be triggered by scattering with electrons and phonons of the substrate (process described by $\hat{J}_{z}$ and $\hat{J}_{ \pm}$operators) or by a transversal magnetic field ( $\hat{J}_{ \pm}$operators) produced for example by an electromagnet or due to the dipolar field generated by the surrounding atoms. These operators induce transitions between levels differing by $\Delta J_{z}=0, \pm 1$ and can induce QTM in nonaxial Kramers doublets. Consequently, Eq. (2) can be extended to

$$
\Delta J_{z}=0 \pm 6 k, \pm 1 \pm 6 k,
$$

where $k$ is an integer varying between 0 and ${ }^{2 J} / 6$. In the case of integer $J$ systems, as already stated by Eq. (2), transitions between the two levels of a doublet are forbidden except for $J_{z}= \pm 3, \pm 6$. In the case of half-integer $J$ systems, transitions between the two levels of a doublet are forbidden only for $J_{z}= \pm^{3} / 2, \pm \frac{9}{2}, \pm 15 / 2$.

Equation (3) applies to both ground and excited doublets. At finite temperature, the energy adsorbed by the atom from substrate electrons and phonons populates excited $J_{z}$ states. Application of Eq. (3) to excited doublets describes thermally activated QTM. We note that, in the case of scattering with phonons, second order transitions with $\Delta J_{z}= \pm 2$ are also possible; however, they were not considered here due to their weak effect.

Distortions to the sixfold symmetry of the graphene CF are possible owing to the nonequivalence of the two carbon sublattices. The strength of these perturbations has been estimated to be of the order of $10^{-7} \mathrm{meV}$ for Dy on graphene/ $\operatorname{Ir}(111)$ [19], hence they are too small to produce relevant effects at $B=0 \mathrm{~T}$. However, they can provide additional relaxation paths in finite fields where different $J_{z}$ levels cross.

\section{EXPERIMENTAL DETAILS}

\section{A. Sample preparation}

The $\operatorname{Ir}(111)$ single crystal was prepared in situ by repeated sputtering and annealing (1400 K) cycles. A single layer of graphene was grown by chemical vapor deposition through exposure of the sample to 100 Langmuir of ethylene at $1400 \mathrm{~K}$ $[6,29]$. RE atoms were deposited from high purity rods $(99.9 \%)$ using an $e$-beam evaporator. Prior to experiments, these rods were degassed until a pressure of $p \leqslant 4 \times 10^{-11}$ mbar during evaporation was reached. RE atoms were deposited on the substrate kept at $4 \mathrm{~K}$.

The coverage of RE atoms is given in monolayers (ML), with one ML being one RE atom per graphene unit cell. For coverage calibration we used STM images of samples partially covered by close-packed RE islands [30]. The density of RE atoms in these islands is given by their respective covalent radii [31]. Thus, from the RE coverage per surface area we infer the equivalent number of RE atoms per graphene unit cell. For these samples characterized with STM, we recorded XAS over the $M_{4,5}$ absorption edges establishing a link between integrated XAS intensity and coverage. We then used XAS as a measure of coverage. 


\section{B. X-ray absorption measurements}

The X-ray absorption measurements were performed at the EPFL/PSI X-Treme beamline at the Swiss Light Source [32]. The experiments were carried out for circularly $\left(\sigma^{+}, \sigma^{-}\right)$and linearly $\left(\sigma^{h}, \sigma^{v}\right)$ polarized X-rays in the total electron yield (TEY) mode at $2.5 \mathrm{~K}$. The XAS corresponds to $\left(\sigma^{+}+\sigma^{-}\right)$, while XMCD and XMLD are defined as $\left(\sigma^{+}-\sigma^{-}\right)$and $\left(\sigma^{h}-\sigma^{v}\right)$, respectively. The measurements were carried out over the $M_{4,5}$ absorption edges of the RE atoms and for two incidence angles with respect to the surface normal, namely normal incidence at $\theta=0^{\circ}$ and grazing incidence at $\theta=60^{\circ}$. In both cases, the magnetic field was collinear with the incident $x$ rays. To take into account the different surface areas illuminated by $\mathrm{x}$ rays in the two geometries, the acquired spectra were normalized with respect to the absorption pre-edge of the corresponding RE element. In addition, the spectra of the clean graphene/Ir(111) surface were acquired prior to the deposition of RE atoms, and were subsequently subtracted from the RE spectra to eliminate any contribution from the substrate. The magnetization curves were acquired by recording the maximum of the XMCD intensity as a function of the external magnetic field for each RE element. The flux of $x$ rays was measured with a photodiode placed after the last optical element of the beamline and is given in units of $\phi_{0}=3 \times 10^{-3}$ photons $\mathrm{nm}^{-2} \mathrm{~s}^{-1}$.

\section{Multiplet calculations}

Multiplet calculations of the RE XAS, XMCD, and XMLD spectra, as well as of the magnetization curves, were performed with the multiX software [33]. The CF generated by the interaction of the RE atom with the neighboring atoms of the graphene/Ir(111) surface was modeled with point charges. This description is justified for the highly localized $4 f$ orbitals of RE atoms due to their mainly electrostatic interaction with the substrate atoms [34-36]. The sign and the value of charges represents the deviation from the free-atom spherical charge distribution surrounding the $4 f$ orbitals. The geometry and strength of these charges were inspired by DFT calculations of single RE atoms on freestanding graphene [24] and adequately tuned for each RE atom. We find that each RE atom on graphene/ $\operatorname{Ir}(111)$ has its unique CF. This indicates that the filling of the $4 f$ orbitals influences the redistribution of the charges at the surface, possibly due to the reduced coordination of the adatoms [24]. This behavior is different from that of RE single-ion molecular magnets, where the $\mathrm{CF}$ is set by the surrounding molecule and replacing one RE ion with another does not cause the redistribution of CF charges, but rather rescales them [37]. The resulting CF parameters are given in the Appendix.

\section{STM measurements}

Scanning tunneling microscopy (STM) measurements were performed with a home-built STM, operating at $5 \mathrm{~K}$ [38]. The images were recorded in constant current mode with W tips. The bias voltage indicated for the STM images refers to the sample.

\section{RESULTS}

\section{A. Dy on graphene/Ir(111)}

As described in our former work [19], Dy atoms on graphene/Ir(111) show magnetic bistability up to $B=5.6 \mathrm{~T}$
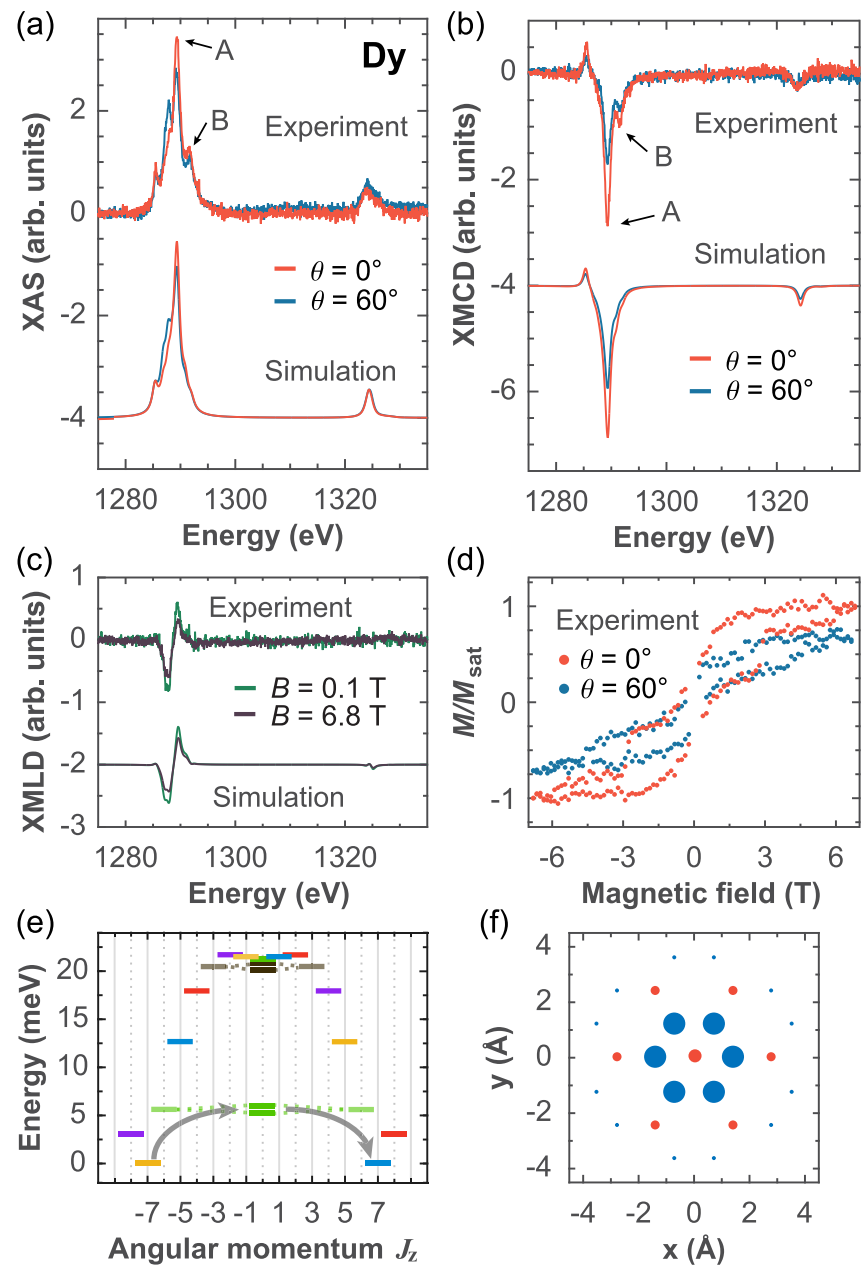

FIG. 1. Measured and simulated (a) XAS, (b) XMCD, and (c) XMLD spectra of $0.004 \mathrm{ML}$ Dy on graphene/ $\operatorname{Ir}(111)(B=6.8 \mathrm{~T}$, $T=2.5 \mathrm{~K}$ ). In (a) and (b), A marks the peak at $1289.3 \mathrm{eV}$, and $\mathrm{B}$ marks the one at $1291.8 \mathrm{eV}$. (d) Magnetization curves obtained by acquiring the XMCD signal at $1289.3 \mathrm{eV}$ as a function of the external field $B\left(T=2.5 \mathrm{~K}, \phi=5 \phi_{0}\right.$ and $\left.\dot{B}=33 \mathrm{mT} \mathrm{s}^{-1}\right)$. (e) Energy diagram of the Dy lowest multiplet $(J=8)$ resulting from multiplet calculations. Each color identifies $J_{z}$ states that belong to one of the six classes of eigenstates defined by the $C_{6 v}$ symmetry. Dashed lines connect doublets with quenched $J_{z}$. Grey arrows indicate the relaxation mechanism at $B=0 \mathrm{~T}$ via thermal excitation to $J_{z}= \pm 6$ doublet. (f) Planar representation of the point charge $\mathrm{CF}$ scheme employed in multiplet calculations; red marks positive, and blue negative charge. The size of each circle reflects the amount of charge. Table II reports spatial coordinates and value of each charge, Table VII the parameters describing the CF splitting in the Stevens operator representation.

at $2.5 \mathrm{~K}$, i.e., they are single-atom magnets. Here we recall their main features and magnetic level structure for the sake of comparison with the ones of other investigated RE elements. Figures 1(a)-1(c) show experimental XAS, XMCD, and XMLD spectra for normal $\left(\theta=0^{\circ}\right)$ and grazing $\left(\theta=60^{\circ}\right)$ $\mathrm{X}$-ray incidence angle for $\mathrm{Dy}$ atoms on graphene/Ir(111). The larger XMCD signal at the $M_{5}$ edge in normal incidence is characteristic of out-of-plane magnetic easy axis. Magnetization curves shown in Fig. 1(d) reveal a wide hysteresis loop (up to $B=5.6 \mathrm{~T}$ for $\theta=0^{\circ}$ ) and magnetic remanence at zero 
field. These features are the fingerprint of long magnetization lifetimes at both zero and at finite fields, i.e., the magnetic ground state is well protected against reversal processes as described in Sec. II. In addition, the wide hysteresis opening in the magnetization curve acquired at $\theta=60^{\circ}$ implies that the magnetic states are stable even under the effect of a strong transverse magnetic field.

We also report the results of the multiplet analysis that has been used to infer the energy level splitting of Dy atoms on graphene/Ir(111) [19]. Simulated absorption spectra are shown in Fig. 1, together with the employed CF scheme and resulting energy diagram. Multiplet calculations reveal divalent Dy monomers on the surface, with the same $4 f$ occupancy as in the gas phase: $4 f^{10}$ with the total angular momentum $J=8$. Further, they reveal $J_{z}= \pm 7$ states as the ground-state doublet. In the $\mathrm{C}_{6 v} \mathrm{CF}$ symmetry, these states are protected against QTM, and scattering with electrons and phonons of the substrate cannot induce direct transitions between them. At $B=0 \mathrm{~T}$, the transition between the levels of the ground-state doublet, and hence the relaxation of the magnetization, can occur only via thermally activated QTM to the first tunnel-split doublet with $J_{z}= \pm 6$, as indicated by grey arrows in Fig. 1(e). This doublet reduces $U_{\text {rev }}$ from $21.4 \mathrm{meV}$ to $5.6 \mathrm{meV}$. Nevertheless, this barrier suffices to ensure magnetic bistability at $2.5 \mathrm{~K}$ and $B=$ $0 \mathrm{~T}$, as demonstrated by the remanence in the magnetization curves [Fig. 1(d)] and long magnetic lifetime of $971 \pm 71 \mathrm{~s}$ [19]. In addition, at finite fields, $U_{\text {rev }}$ essentially coincides with the full value of TZFS. This further protects the ground state against thermally activated QTM and allows for magnetic hysteresis up to $5.6 \mathrm{~T}$. Level crossings of lowest lying quantum states prevent magnetic bistability at higher fields [19].

\section{Coverage dependence of Dy XAS spectra}

The XAS and XMCD spectra of Dy monomers on graphene/ $\operatorname{Ir}(111)$ are characterized by the pronounced peak at $1289.3 \mathrm{eV}$ (peak A). However, in the experimental spectra there is an additional peak at $1291.8 \mathrm{eV}$ (peak B) which is not reproduced in simulations [Figs. 1(a) and 1(b)]. This peak indicates the presence of Dy $4 f^{9}$ occupancy [39] and can be associated with the formation of Dy dimers. To verify this assignment, and to further investigate the influence of dimers and bigger clusters on the magnetic properties of the ensemble, we performed combined coverage dependent XAS and STM measurements. Figure 2(a) shows the Dy $M_{5}$ edge as a function of coverage between $0.004 \mathrm{ML}$ and $0.032 \mathrm{ML}$. The peak B is already faintly present for coverages as low as $0.004 \mathrm{ML}$, and its intensity increases with increasing Dy coverage. At 0.032 ML, this peak reaches the intensity of the Dy monomer's peak A. The increasing intensity of peak B and of its tail also result in the shift of peak A toward higher energies. From this coverage dependence we attribute the peak B to the formation of Dy dimers and bigger clusters, and the corresponding change in the valency to the increased coordination of the Dy atoms [39].

To corroborate this assignment, we performed STM measurements of the mean cluster size as a function of coverage. Figures 2(b)-2(e) show STM images for Dy coverages comparable to the ones employed in the XAS measurements. Dysprosium monomers appear as protrusions with large apparent height and perfectly circular shape, while dimers have smaller apparent height with oblate shape [19]. Figure 2(f) shows the coverage dependence of the mean Dy cluster size

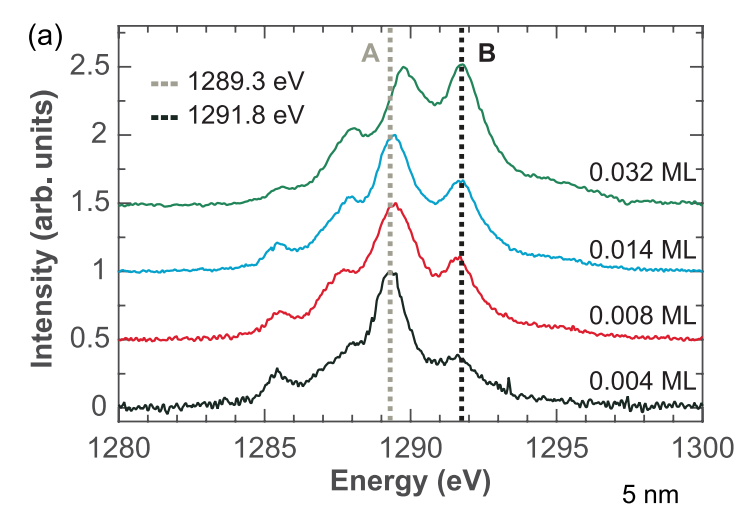

(b)
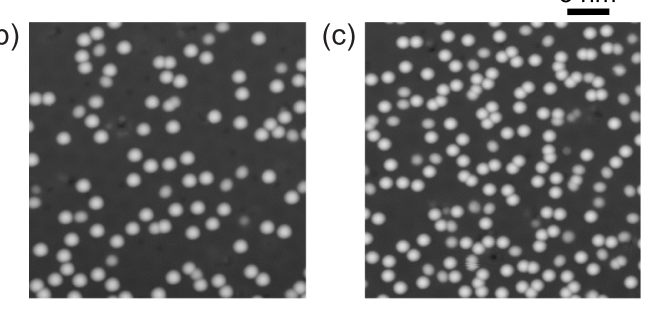

(d)

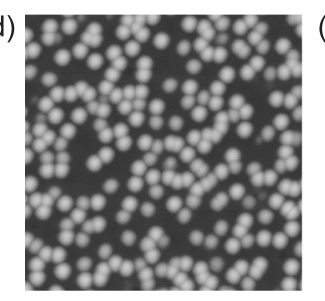

(e)

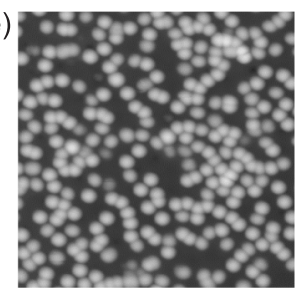

(f)

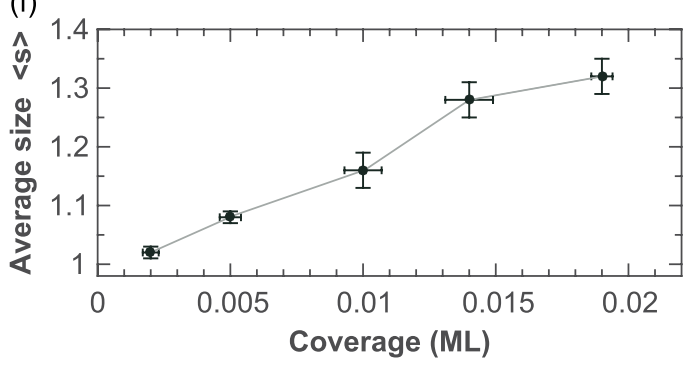

FIG. 2. (a) Coverage dependence of Dy XAS at the $M_{5}$ edge acquired at $\theta=0^{\circ}$ and $B=0.05 \mathrm{~T}$. All spectra were normalized to the intensity at the peak at or near $1289.3 \mathrm{eV}$, and were subsequently offset for clarity. Dashed lines mark the peak energy associated with divalent Dy atoms at $1289.3 \mathrm{eV}$ (grey) and trivalent Dy clusters at $1291.8 \mathrm{eV}$ (black). STM images for coverages close to the ones employed in XAS measurements: (b) $0.005 \mathrm{ML}$, (c) $0.010 \mathrm{ML}$, (d) $0.014 \mathrm{ML}$, and (e) $0.019 \mathrm{ML}$. The scale bar applies to all images [(b)-(d): $V_{\mathrm{t}}=-0.5 \mathrm{~V}$, $\left.I_{\mathrm{t}}=20 \mathrm{pA},(\mathrm{e}): V_{\mathrm{t}}=-0.2 \mathrm{~V}, I_{\mathrm{t}}=100 \mathrm{pA} ; T_{\text {dep }}=10 \mathrm{~K}, T=5 \mathrm{~K}\right]$. (f) Coverage dependence of Dy average cluster size deducted from STM measurements. Grey line serves as guide to the eye.

$\langle s\rangle$, which increases from 1.02 for 0.002 ML (STM image not shown), to 1.32 for 0.019 ML. This size dependence, and hence the dimer abundance is inconsistent with statistical growth [40]. However, it can be explained by the large direct impingement area of the already adsorbed Dy atoms, similar to what has been observed for $\mathrm{Er}$ on $\mathrm{Cu}(111)$ [31].

\section{Time evolution of Dy XAS spectra}

In addition to the change in shape of Dy XAS with increasing coverage, we observe a similar change with exposure 


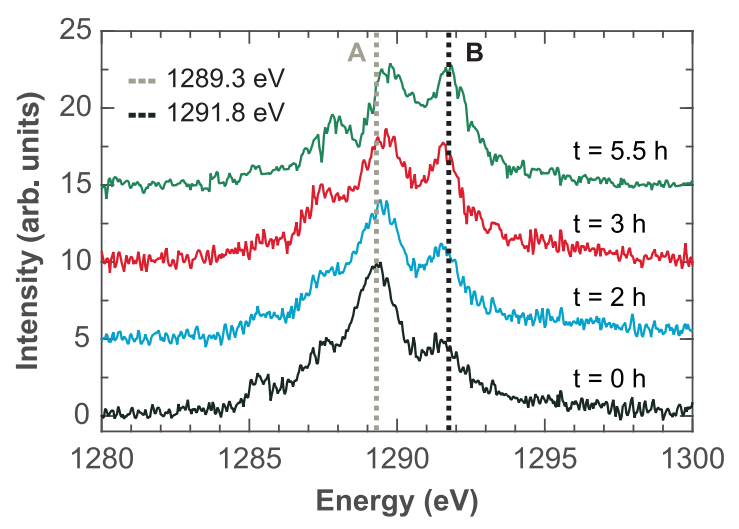

FIG. 3. Exposure time dependence of the XAS for 0.01 ML Dy on graphene/ $\operatorname{Ir}(111)$ at the $M_{5}$ edge. All spectra were acquired at $\theta=0^{\circ}$ and $B=6.8 \mathrm{~T}$. Dashed lines mark the peak positions associated with divalent Dy atoms at $1289.3 \mathrm{eV}$ (grey) and trivalent Dy objects at $1291.8 \mathrm{eV}$ (black). Spectra are vertically offset for clarity.

time to the residual gas of the UHV cryostat. Figure 3 shows four spectra at the Dy $M_{5}$ edge acquired during the course of five and a half hours of measurements. Initially, right after the deposition of the Dy atoms, the spectrum is characterized by a pronounced peak $\mathrm{A}$, and a very small peak $\mathrm{B}$. As the exposure time increases, the intensity of peak B increases and, in turn, causes a shift of the peak A toward higher energies. The divalent $R E$ atoms on surfaces are known to be highly reactive and sensitive to contamination [41]. Hence, we associate this change in the spectra to the contamination of Dy monomers with molecules from the residual gas in the cryostat, most likely atomic hydrogen after the dissociative adsorption of $\mathrm{H}_{2}$ [5].

\section{Flux dependence of Dy magnetization curves}

The magnetic relaxation time has been reported to depend on x-ray photon flux $[17,42,43]$. This effect is due to the generation of secondary electrons that destabilize the magnetic quantum states. When the magnetic lifetime becomes comparable to the acquisition time of the each point in the magnetization curve, narrowing of the hysteresis loop is observed [17]. Figure 4 shows two magnetization curves of

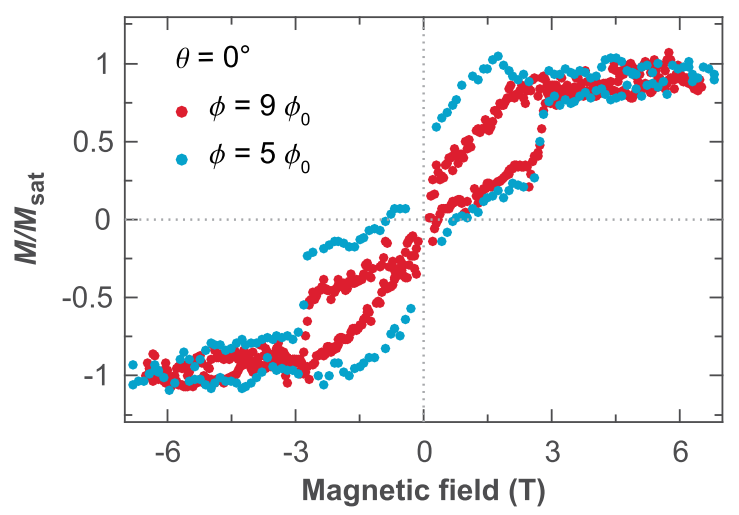

FIG. 4. Magnetization curves of Dy on graphene/Ir(111) acquired with two combinations of x-ray flux and acquisition speeds $(T=$ $2.5 \mathrm{~K}, \theta=0^{\circ}$; blue, $\phi=5 \phi_{0}$ and $\dot{B}=33 \mathrm{mT} \mathrm{s}^{-1}$; red, $\phi=9 \phi_{0}$, and $\dot{B}=12 \mathrm{mT} \mathrm{s}^{-1}$ ).
Dy on graphene/Ir(111) acquired with two sets of x-ray fluxes and acquisition speeds. The curve measured at $\phi=5 \phi_{0}$ and $\dot{B}=33 \mathrm{mT} \mathrm{s}^{-1}$ shows prominent remanence, while the one acquired with nearly twice the flux and one-third of the speed is essentially closed at $B=0 \mathrm{~T}$. Overall, the entire hysteresis has become narrower. This confirms that, at the employed flux and field sweep speed, the magnetic lifetime of Dy atoms on graphene/Ir(111) are comparable to the one between successive points in the magnetization curve. Evidently, for very fast or very slow magnetic relaxation with respect to the acquisition time, the magnetization curves are much less sensitive to the measurement parameters. In those cases, the flux and field sweep can be optimized for best signal-to-noise ratio.

\section{B. Divalent RE atoms on graphene/Ir(111)}

In the following, we present the results obtained for other $\mathrm{RE}$ atoms adsorbed on the graphene/Ir(111) surface that show the divalent $4 f$ configuration of the free atom. We studied both early and late lanthanide elements, as well as integer and half-integer $J$ systems.

Nd on graphene/Ir(111). In their divalent configuration, $\mathrm{Nd}$ atoms have four electrons in their $4 f$ orbitals and represent the counterpart to Dy atoms, which have four holes. Therefore, $\mathrm{Nd}$ and Dy have the same values of spin and orbital moments while, due to the opposite sign of the spin-orbit coupling in early and late lanthanides, the corresponding $J$ values are different. To investigate the magnetic properties of $\mathrm{Nd}$ atoms on graphene/Ir(111), we acquired their XAS, XMCD, and XMLD spectra at the $M_{4,5}$ edges, see Figs. 5(a)-5(c). We observe pronounced spectral features at both adsorption edges. In XMCD spectra, these features have opposite sign, with the $M_{5}$ edge being overall positive and the $M_{4}$ edge negative [Fig. 5(b)]. Higher intensity of the XMCD signal in normal with respect to grazing incidence of $\mathrm{x}$ rays indicates out-of-plane easy magnetization axis of this system. Since the $\mathrm{XMCD}$ intensity is largest at the $M_{4}$ edge, we acquired magnetization curves of $\mathrm{Nd}$ atoms at its peak energy of $996.7 \mathrm{eV}$ for both normal and grazing incidence. The results are shown in Fig. 5(d). Both curves show paramagnetic behavior at $2.5 \mathrm{~K}$. The magnetization curve recorded in normal incidence is much steeper around $B=0 \mathrm{~T}$ and saturates for $B \geqslant|4| \mathrm{T}$, whereas the magnetization curve acquired at in grazing incidence reaches a lower saturation value at the highest available field. This is an additional evidence of an out-of-plane easy axis.

Further insight into the electronic structure and magnetic ground state of $\mathrm{Nd}$ atoms on graphene/ $\operatorname{Ir}(111)$ is provided by multiplet calculations. We mainly focus on reproducing three key features that determine the energy diagram of this system. These are the spectral features of the XAS, XMCD, and XMLD spectra, the overall shape of the magnetization curves [Fig. 5(d)], and the ratio of intensities of the XMCD peaks in normal and grazing incidence at $B=6.8 \mathrm{~T}$ [Fig. 5(b)]. This ratio sets the relative amplitude of the corresponding magnetization curves at the same field and it amounts to approximately $1 / 2$. This means that the XMCD intensity rescales as the cosine of the $\mathrm{x}$-ray incidence angle $\theta$, suggesting maximal $J_{z}$ as magnetic ground state and large splitting of the magnetic levels. We obtained the best fit to the experiment using the point charge CF scheme shown in Fig. 5(f). All the spectral features observed in the experiments are well reproduced, except for the 

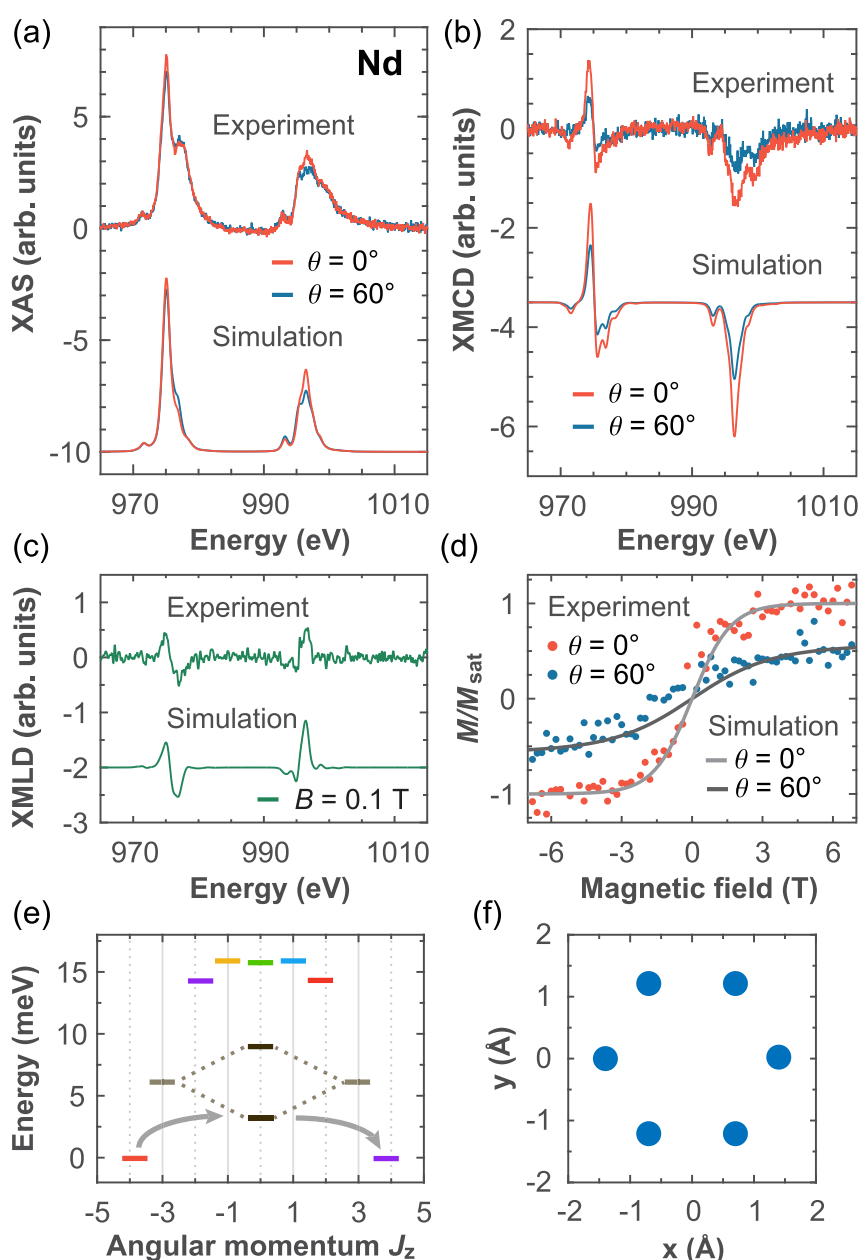

(d)

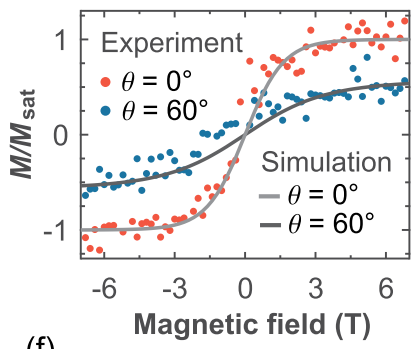

(f)

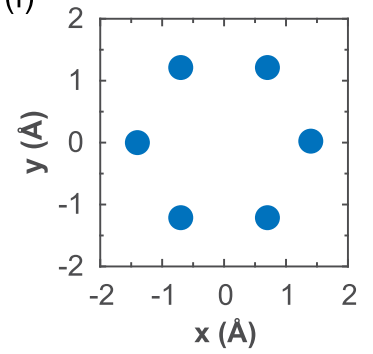

FIG. 5. Experimentally acquired and simulated (a) XAS, (b) XMCD, and (c) XMLD spectra of $0.002 \mathrm{ML} \mathrm{Nd}$ on graphene/ $\operatorname{Ir}(111)(B=6.8 \mathrm{~T}, T=2.5 \mathrm{~K})$. (d) Magnetization curves obtained by acquiring the XMCD signal at $996.7 \mathrm{eV}(T=$ $2.5 \mathrm{~K}, \phi=9 \phi_{0}$ and $\dot{B}=12 \mathrm{mT} \mathrm{s}^{-1}$ ), together with simulated ones. (e) Energy diagram of the lowest multiplet $(J=4)$ resulting from multiplet calculations. Each color identifies $J_{z}$ states that belong to one of the six classes of eigenstates defined by the $C_{6 v}$ symmetry. Dashed lines connect $J_{z}= \pm 3$ doublet with quenched $\left\langle J_{z}\right\rangle$. Grey arrows indicate relaxation mechanism at $B=0 \mathrm{~T}$ via thermal excitation to $J_{z}= \pm 3$ doublet. (f) Planar representation of the point charge $\mathrm{CF}$ scheme employed in multiplet calculations; blue marks negative charge. Table III reports spatial coordinates and value of each charge, Table VII the parameters describing the CF splitting in the Stevens operator representation.

peak at $977.3 \mathrm{eV}$. Similarly to what was observed for Dy, this disagreement can be attributed to clusters and/or contaminated atoms; nevertheless, they provide a minor contribution to the properties of the ensemble. Multiplet calculations reveal $4 f^{4}$ occupation with $J=4$, as for $\mathrm{Nd}$ atoms in the gas phase. Similar occupation of nearly four electrons in their $4 f$ orbitals has been calculated by DFT for $\mathrm{Nd}$ atoms on freestanding graphene [20]. The energy diagram resulting from multiplet calculations is shown in Fig. 5(e).

The adsorption of $\mathrm{Nd}$ in the hollow site of graphene results in a strong uniaxial anisotropy with the maximal $J_{z}= \pm 4$ magnetic ground state, as anticipated from the ratio of XMCD intensities at $B=6.8 \mathrm{~T}$. The TZFS is approximately $16 \mathrm{meV}$; however, the lowest lying excited states with $J_{z}= \pm 3$ provide a shortcut for the thermally activated magnetization relaxation. These levels are strongly mixed and tunnel-split in the $C_{6 v}$ CF symmetry of graphene. The resulting pair of singlet states with quenched magnetic moment are separated by $\Delta_{-3,3}=$ $5.9 \mathrm{meV}$, with the lower of the two being at the energy of $3.2 \mathrm{meV}$. The large separation $\Delta_{-3,3}$ provides a substantially larger QTM rate with respect to the Dy case [44]. Hence, this tunnel-split doublet strongly reduces $U_{\text {rev }}$ and is responsible for the lack of magnetic hysteresis in $\mathrm{Nd}$ atoms Fig. 5(d). The thermally activated shortcut for magnetization reversal is indicated with grey arrows in Fig. 5(e).

Ho on graphene/Ir(111). In the divalent state, Ho atom has an additional electron in the $4 f$ orbitals with respect to divalent Dy, resulting in a half integer value of $J$. To investigate the magnetic properties of these atoms on graphene/ $\operatorname{Ir}(111)$, we acquired XAS, XMCD, and XMLD spectra over the Ho $M_{4,5}$ edges, see Figs. 6(a)-6(c). Differently from Dy and Nd, Ho atoms exhibit a slightly larger XMCD signal in grazing incidence [Fig. 6(b)], hence indicating a weak in-plane anisotropy. Given the larger intensity of the absorption and dichroism at the $M_{5}$ edge, we acquired the magnetization curves at the $M_{5}$ peak energy of $1346.6 \mathrm{eV}$ [Fig. 6(d)]. The magnetization curve recorded in normal incidence is steeper around $B=0 \mathrm{~T}$ and saturates already at about $B=2 \mathrm{~T}$. However, the curve obtained in grazing incidence crosses it at $B=3 \mathrm{~T}$, and it does not saturate even at the highest available magnetic field of $B=6.8 \mathrm{~T}$. This peculiar behavior suggests a ground state with intermediate value of $J_{z}$ that can produce large projected magnetic moments along both directions of incidence.

Further insight is again provided by comparing the data with multiplet calculations. We focused on reproducing the spectral features of Ho shown in Figs. 6(a)-6(c), with particular emphasis on (i) the peculiar crossover between the normal and grazing incidence magnetization curves [Fig. 6(d)] and (ii) the distinct behavior of the XMLD spectra [Fig. 6(c)] whose intensity increases with increasing magnetic field. Reproducing these features requires the use of a complex point charge $\mathrm{CF}$ scheme, as shown in Fig. 6(f). Our simulations reveal 11 electrons in the Ho $4 f$ orbitals, a lowest multiplet with $J=15 / 2$, and a TZFS of $23 \mathrm{meV}$. The resulting energy diagram is shown in Fig. 6(e). The ground-state doublet of this system has $\left\langle J_{z}\right\rangle= \pm 4.3$, i.e., very close to the pure $J_{z}= \pm^{9} / 2$ ground state. Note that the distinctive behavior of XMLD spectra is unique to it and it could not be reproduced by any other ground-state doublet in our calculations. This ground state should, in principle, result in a stable magnetization of this system; nevertheless, the absence of hysteresis in magnetization curves [Fig. 6(d)] indicates a magnetic lifetime of order of seconds or less at $2.5 \mathrm{~K}$.

Given the large TZFS of $23 \mathrm{meV}$, thermally activated processes overcoming the full barrier must be excluded. Therefore, relaxation has to occur via thermally activated QTM involving nearest excited states. At $B=0 \mathrm{~T}$, the lowest lying excited states are $J_{z}= \pm^{5} / 2$ at $1.6 \mathrm{meV}$, which cannot be reached by first-order transitions. Hence, the lowest lying levels that participate in scattering processes with $\Delta J_{z}= \pm 1$ are $\left\langle J_{z}\right\rangle=$ \pm 5.4 , close to the pure $J_{z}= \pm{ }^{11} / 2$ doublet and located at 

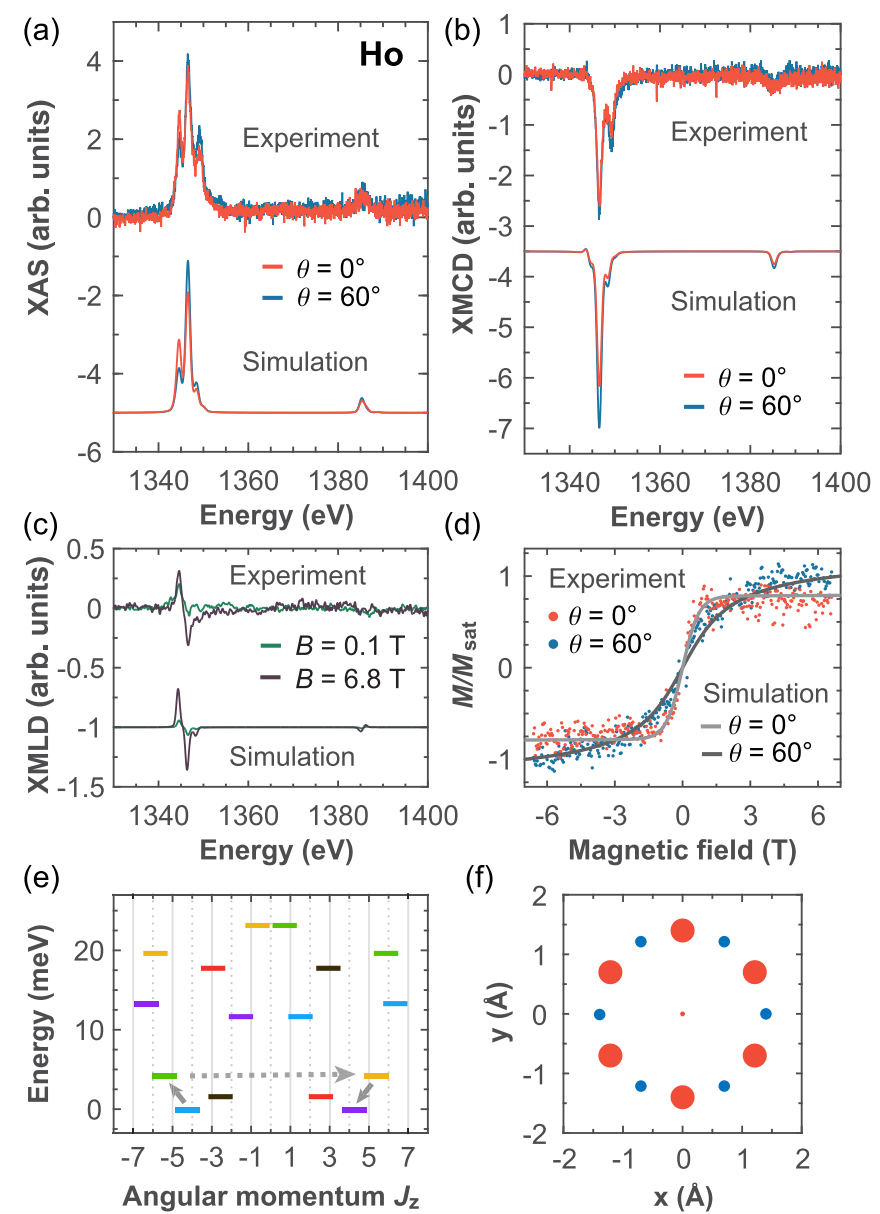

FIG. 6. Experimentally acquired and simulated (a) XAS, (b) XMCD, and (c) XMLD spectra of 0.003 ML Ho on graphene/Ir(111) ( $B=6.8 \mathrm{~T}, T=2.5 \mathrm{~K})$. (d) Magnetization curves obtained by acquiring the XMCD signal at $1346.6 \mathrm{eV}\left(T=2.5 \mathrm{~K}, \phi=9 \phi_{0}\right.$ and $\dot{B}=12 \mathrm{mT} \mathrm{s}^{-1}$ ), together with simulated ones. (e) Energy diagram of the Ho lowest multiplet $(J=15 / 2)$, resulting from multiplet calculations. Each color identifies $J_{z}$ states that belong to one of the six classes of eigenstates defined by the $C_{6 v}$ symmetry. Grey arrows indicate the relaxation mechanism at $B=0 \mathrm{~T}$ via thermal excitation to $\left\langle J_{z}\right\rangle= \pm 5$.4. (f) Planar representation of the point charge $\mathrm{CF}$ scheme employed in multiplet calculations; red marks positive and blue negative charge. The size of each circle reflects the amount of charge. Table IV reports spatial coordinates and value of each charge, Table VII the parameters describing the CF splitting in the Stevens operator representation.

$4.2 \mathrm{meV}$ [see solid-line arrows in Fig. 6(e)]. This doublet is a nonaxial one, thus, according to Eq. (3), QTM can occur between its levels [indicated by dashed lines in Fig. 6(e)].

Er on graphene/Ir(111). Er atoms on graphene/ $\operatorname{Ir}(111)$ in their divalent configuration have one $4 f$ electron more than Ho. The corresponding XAS, XMCD and XMLD spectra are shown in Fig. 7. The considerably larger XMCD intensity in grazing with respect to normal incidence [Fig. 7(b)] indicates strong in-plane anisotropy, and implies a low $J_{z}$ ground state. For these atoms, the high sensitivity to contamination strongly limited the time frame for a meaningful experiment, thus hindering the acquisition of the magnetization curves. The lack of magnetization curves limits the precision of the energy level
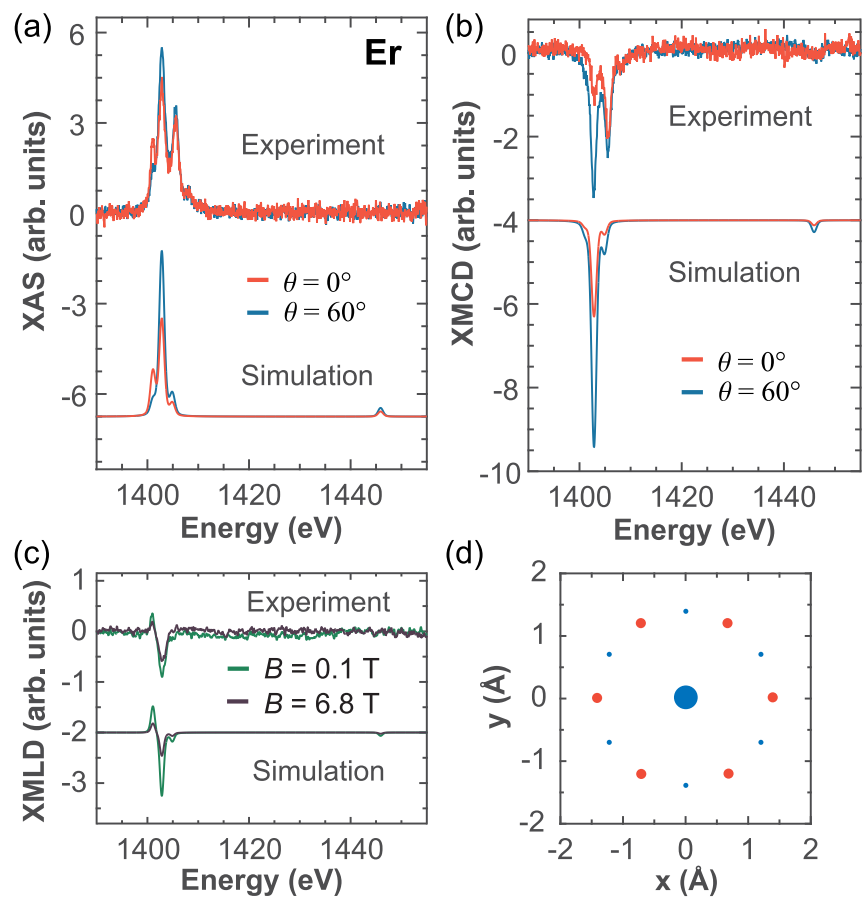

(d)

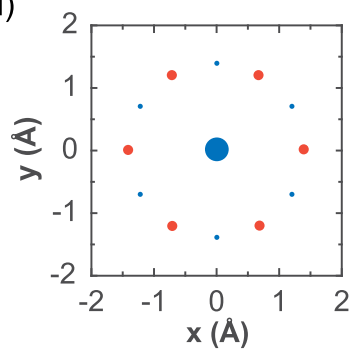

(e)

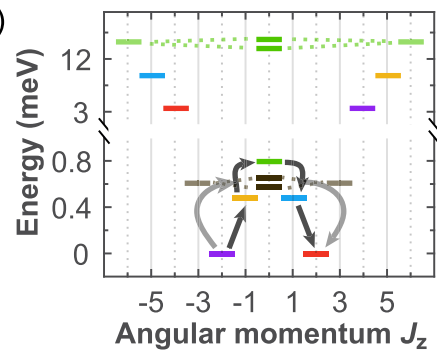

FIG. 7. Experimentally acquired and simulated (a) XAS, (b) XMCD, and (c) XMLD spectra of 0.004 ML Er on graphene/Ir(111) ( $B=6.8 \mathrm{~T}, T=2.5 \mathrm{~K})$. (d) Planar representation of the point charge $\mathrm{CF}$ scheme employed in multiplet calculations; red marks positive and blue negative charge. The size of each circle reflects the amount of charge. Table V reports spatial coordinates and value of each charge, Table VII the parameters describing the CF splitting in the Stevens operator representation. (e) Energy diagram of the Er lowest multiplet $(J=6)$. Each color identifies $J_{z}$ states that belong to one of the six classes of eigenstates defined by the $C_{6 v}$ symmetry. Dashed lines connect doublets with quenched $\left\langle J_{z}\right\rangle$. Grey and black arrows indicate two relaxation mechanisms at $B=0 \mathrm{~T}$.

splitting inferred from the multiplet calculations. Nevertheless, we performed calculations focusing on reproducing (i) the spectral shape of XAS, XMCD, and XMLD, (ii) the in-plane anisotropy of this system with the adequate ratio of XMCD peaks in normal and grazing incidence at $6.8 \mathrm{~T}$ [Fig. 7(b)], and (iii) the higher intensity of XMLD at $0.1 \mathrm{~T}$, than at $6.8 \mathrm{~T}$.

The spectra resulting from multiplet calculations performed using the point charge CF scheme shown in Figs. 7(d) are shown in Figs. 7(a)-7(c). They reproduce well all the spectral features observed in experiments apart from the peak at 1405.6 $\mathrm{eV}$ [see Figs. 7(a) and 7(b)] whose intensity is much higher in the experimental spectra. Similarly to the other RE elements, we attribute this peak to clusters and/or contaminated Er atoms in the ensemble. Since our simulations do not take these 
objects into account, their features are not reproduced. Our calculations reveal $4 f^{12}$ occupation and a lowest multiplet with $J=6$, as for Er atoms in gas phase. The resulting energy diagram is shown in Fig. 7(e). The TZFS of this system is $15 \mathrm{meV}$ and its ground state is the $J_{z}= \pm 2$ doublet. In addition, there are several levels with $J_{z} \leqslant 3$ within less than $1 \mathrm{meV}$, with the tunnel-split $J_{z}= \pm 3$ levels lying at $0.6 \mathrm{meV}$ above the ground-state doublet.

The very small energy separation between the lowest lying levels facilitates magnetization reversal through scattering with electrons and phonons of the substrate. Thus, no magnetic bistability is expected for this system. At $B=0 \mathrm{~T}$, there are two main magnetization reversal paths. In the first, the relaxation occurs via thermal excitation to the tunnel-split $J_{z}= \pm 3$ states at $0.6 \mathrm{meV}$. In the second, the relaxation path consists of thermal excitation to $J_{z}=0$ via the $J_{z}= \pm 1$ states. The two magnetization reversal mechanisms are indicated in Fig. 7(e) with grey and black arrows, respectively.

\section{Trivalent RE atoms on graphene/ $\operatorname{Ir}(111)$}

Tb on graphene/Ir(111). Experimentally acquired XAS, $\mathrm{XMCD}$, and XMLD spectra for $\mathrm{Tb}$ on graphene/ $\operatorname{Ir}(111)$ are shown in Figs. 8(a)-8(c). Unlike for the other lanthanide atoms, the XAS spectral features of $\mathrm{Tb}$ atoms indicate a trivalent configuration of these atoms on the graphene/Ir(111) surface. This $4 f^{8}$ configuration is characterized by a double-peak structure in the Tb $M_{5}$ edge $[45,46]$. The higher intensity of the XMCD signal acquired in grazing with respect to normal incidence indicates an in-plane anisotropy. Similarly to the other late lanthanide elements, we acquired magnetization curves at the peak energy of the $M_{5}$ edge that corresponds to the maximum XMCD intensity. The resulting curves for both normal and grazing incidence of $x$ rays are shown in Fig. 8(d). Both curves show paramagnetic behavior at $2.5 \mathrm{~K}$.

Following the same procedure adopted for the other elements, we performed multiplet calculations to access the magnetic level structure of $\mathrm{Tb}$ atoms on graphene/Ir(111). For this case, we focused on reproducing (i) the in-plane anisotropy with the appropriate ratio of XMCD intensities for the two incidences of $x$ rays at $6.8 \mathrm{~T}$ [Fig. 8(b)] and (ii) the peculiar shape of the XAS for $\theta=0^{\circ}$. We obtained the best agreement with the experimental spectra using the point charge CF scheme shown in Fig. 8(f). The resulting spectra overall reproduce the main features of the experimental ones. However, the intensities of the XAS for $\theta=60^{\circ}$, as well as the intensities of XMCD spectra for both incidences of $x$ rays are not well reproduced. Better matching of these intensities leads to poor matching of the shape and the ratio of the magnetization curves. In agreement with our expectations based on the spectral shape of the XAS, our multiplet calculations reveal trivalent configuration with eight electrons in Tb's $4 f$ orbitals. The lowest multiplet of magnetic states has $J=6$ and its energy diagram is shown in Fig. 8(e).

The resulting TZFS of $7 \mathrm{meV}$ is the smallest among all the lanthanide atoms in this study. The ground state of this system consists of a tunnel-split doublet with $J_{z}= \pm 3$. These states are strongly mixed and tunnel-split in the $\mathrm{C}_{6 v} \mathrm{CF}$ of graphene resulting in a doublet of quenched $J_{z}$ for which QTM occurs. This ground state is responsible for the lack
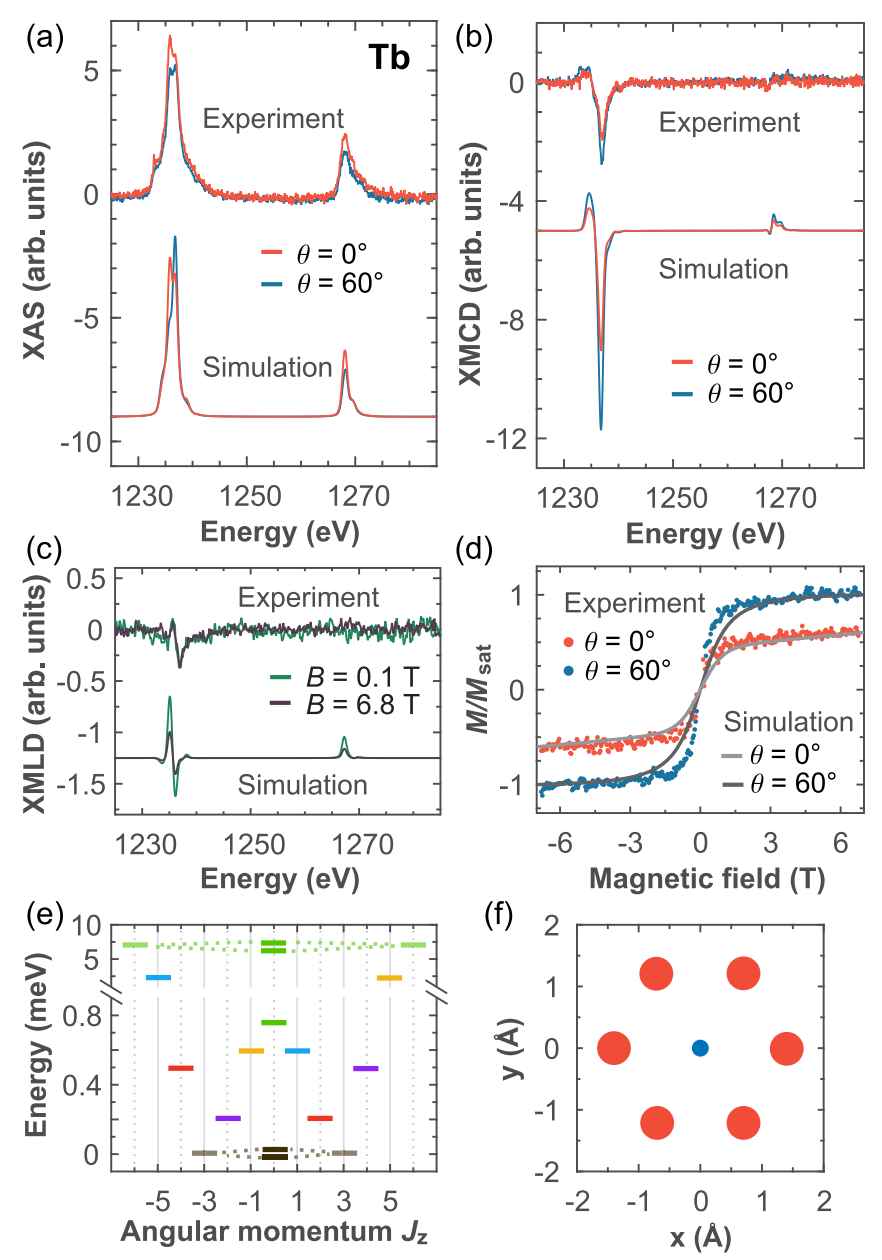

FIG. 8. Experimentally acquired and simulated (a) XAS, (b) XMCD, and (c) XMLD spectra of $0.003 \mathrm{ML}$ Tb on graphene/Ir(111) $(B=6.8 \mathrm{~T}, T=2.5 \mathrm{~K})$. (d) Magnetization curves obtained by acquiring the XMCD signal at $1236.9 \mathrm{eV}\left(T=2.5 \mathrm{~K}, \phi=9 \phi_{0}\right.$ and $\dot{B}=12 \mathrm{mT} \mathrm{s}^{-1}$ ), together with simulated ones. (e) Energy diagram of the Tb lowest multiplet $(J=6)$ resulting from multiplet calculations. The splitting at $J_{z}=0$ is emphasized for better clarity. Each color identifies $J_{z}$ states that belong to one of the six classes of eigenstates defined by the $C_{6 v}$ symmetry. Dashed lines connect doublets with quenched $\left\langle J_{z}\right\rangle$. (f) Planar representation of the point charge CF scheme employed in multiplet calculations; red marks positive and blue negative charge. The size of each circle reflects the amount of charge. Table VI reports spatial coordinates and value of each charge, Table VII the parameters describing the CF splitting in the Stevens operator representation.

of magnetic remanence in $\mathrm{Tb}$ atoms at $B=0 \mathrm{~T}$, whereas for $B \neq 0 \mathrm{~T}$ the small energy separation between other magnetic levels enables efficient magnetization reversal by scattering with electrons and phonons of the substrate, hence hindering magnetic hysteresis [see Fig. 8(d)].

\section{DISCUSSION}

\section{A. $4 \boldsymbol{f}$ occupation of $\mathrm{RE}$ atoms on graphene/Ir(111)}

The electronic configuration and $4 f$ occupancy of lanthanide atoms on a supporting substrate is ruled by two 
TABLE I. Occupation of $4 f$ orbitals $n$, total angular momentum $J$, effective ground state $\left\langle J_{z}\right\rangle$ value, $U_{\text {rev }}$ barrier and lifetime $\tau$ at $B=0 \mathrm{~T}$ for RE atoms on graphene/Ir(111).

\begin{tabular}{lrcccc}
\hline \hline Element & $n$ & $J(\hbar)$ & $\left\langle J_{z}\right\rangle(\hbar)$ & $U_{\text {rev }}(\mathrm{meV})$ & $\tau(\mathrm{s})$ \\
\hline $\mathrm{Dy}$ & 10 & 8 & \pm 7 & 5.6 & $971^{\mathrm{a}}$ \\
$\mathrm{Nd}$ & 4 & 4 & \pm 4 & 3.2 & 0.01 \\
$\mathrm{Ho}$ & 11 & $15 / 2$ & \pm 4.3 & 4.2 & 1.3 \\
$\mathrm{Er}^{\mathrm{b}}$ & 12 & 6 & \pm 2 & $<1$ & $<5 \times 10^{-7}$ \\
$\mathrm{~Tb}$ & 8 & 6 & \pm 3 & 0 & 0 \\
\hline \hline
\end{tabular}

a Measured value [19].

${ }^{\mathrm{b}} U_{\text {rev }}$ value is less accurate for Er, as we could not record magnetization curves for that system.

competing quantities, namely the energy needed to change from a divalent $\left(4 f^{n}\right)$ to a trivalent $\left(4 f^{n-1}\right)$ atomic configuration and the difference in their surface binding energies. The former is given as a sum of the $f-d$ promotion energy $E_{f d}$ and the intershell coupling energy $\Delta E_{c}$, called atomic correction energy $[47,48]$. The surface binding energy strongly depends on the atom's environment as it is ruled by the hybridization between its outer $s p d$ orbitals with the valence electrons of the substrate. The degree of hybridization, and hence the binding energy, increases with the increasing substrate density of states (DOS) at $E_{F}$ [39].

When atoms are placed on substrates with low DOS at $E_{F}$, such as graphene, the atomic correction energy is the leading term. Among the lanthanide atoms studied, this energy is the smallest for $\mathrm{Tb}$, amounting to only $0.49 \mathrm{eV}$, while for all other lanthanide atoms it is about three times larger [47]. The valency of lanthanide atoms on graphene follows this trend in atomic correction energy. The only lanthanide found in trivalent configuration is $\mathrm{Tb}$, whereas all the other ones are in their divalent configuration. In addition, it has been demonstrated that the divalent state of $\mathrm{Tb}$ is highly unfavorable even for freestanding dimers, i.e., a single metallic bond is sufficient to trigger its trivalent configuration [48]. Hence, a Tb atom on graphene takes on the most common trivalent configuration that is also observed for $\mathrm{TbPc}_{2}$ molecular magnets [43,49].

The valency of divalent lanthanide atoms is highly sensitive to their coordination. An increase in coordination, either via cluster nucleation or attachment of contaminants, induces a trivalent configuration in these atoms, as it has been demonstrated in Sections IV A 1 and IV A2. In the case of cluster nucleation, valence bands are formed, hence enabling the transfer of an electron from the $4 f$ to the spd bands [48]. Trivalent lanthanide atoms, on the other hand, keep their valency unchanged even upon an increase in coordination, as it has been demonstrated for these atoms on metal substrates [31,39] and Tb atoms on graphene/Ir(111).

\section{B. Magnetic stability of $\mathrm{RE}$ atoms on graphene/Ir(111)}

The quantum level structure of lanthanide atoms contributes to determine their magnetic lifetime. We find large TZFS with a ground state that is protected against QTM for Nd, Dy, and Ho. However, only Dy shows hysteresis in its magnetization curves, with significant magnetic stability on the timescale of our experiments at $2.5 \mathrm{~K}$. In first approximation, missing a precise quantification of the timescale associated with the different spin-reversal mechanisms, these observations can be rationalized considering the different heights of $U_{\text {rev }}$, which are given by the first excited states enabling thermally activated QTM. The values of $U_{\text {rev }}$ inferred from our multiplet calculations are summarized in Table I. Out of the systems considered, Dy indeed shows the highest value of $U_{\text {rev }}=$ $5.6 \mathrm{meV}$. For Dy and Nd, QTM proceeds through the first tunnel-split doublet. However, in the case of $\mathrm{Nd}$, a smaller $U_{\text {rev }}$ barrier and a substantially larger separation between the $J_{z}= \pm 3$ levels with respect to Dy $J= \pm 6$ ones result in lack of magnetic stability at $2.5 \mathrm{~K}$. In the case of Ho, QTM is activated at the lowest states that are mixed with $J_{z}= \pm^{1 / 2}$. For $\mathrm{Er}$ atoms, magnetization reversal between $J_{z}= \pm 2$ ground states is easily achieved by thermal transitions to the lowest excited states situated at less than $1 \mathrm{meV}$. This energy separation sets an upper bound of $U_{\text {rev }}$ for this system. Finally, in the case of Tb, the lack of magnetic stability is due to its tunnel-split ground state doublet. This ground state supports QTM and hinders any magnetic stability.

The zero-field magnetic lifetime of RE on graphene/Ir(111) can be quantitatively evaluated on the basis of $U_{\text {rev }}$ using an Arrhenius law describing the magnetization reversal:

$$
\tau=\tau_{0} \exp \left(\frac{U_{\mathrm{rev}}}{k_{\mathrm{B}} T}\right)
$$

where $\tau$ is the magnetic lifetime of a system at temperature $T$ and employed x-ray flux $\phi, \tau_{0}$ is the characteristic time between two consecutive reversal attempts and $k_{\mathrm{B}}$ is the Boltzmann constant. $\tau_{0}$ can be estimated for Dy on graphene/Ir(111) using the measured magnetic lifetime of $971 \mathrm{~s}$ at $2.5 \mathrm{~K}$ and $U_{\text {rev }}$ of $5.6 \mathrm{meV}$. Since the measured $\tau$ depends on the photon flux, the value of $\tau_{0, \text { eff }}=4.5 \times 10^{-9} \mathrm{~s}$ obtained from the experimental data represents the lower bound for the intrinsic $\tau_{0}$. Extrapolating $\tau$ at zero photon flux from flux dependent measurements [19] gives a value of about $2500 \mathrm{~s}$, suggesting that the intrinsic value of $\tau_{0}$ does not exceed $3 \tau_{0 \text {,eff }}$. This value is comparable to the ones obtained for the $\mathrm{Fe}_{4}$ molecular magnets adsorbed on a metal surface [50] and on graphene [51]. Assuming that $\tau_{0}$ is primarily dependent on the properties of the substrate and specifically of its phonons, we can use the same value to estimate the magnetic lifetime of the other RE atoms. Taking their $U_{\text {rev }}$ values, we calculated the lifetime of each RE system. The obtained values are given in Table I. As expected from considerations about their $U_{\text {rev }}$, the only system besides Dy that could show considerable magnetic lifetime at $2.5 \mathrm{~K}$ is Ho with $1.3 \mathrm{~s}$. Nevertheless, this lifetime is shorter than the time needed for the acquisition of a single point in our magnetization curves, which explains the lack of hysteresis in our measurements.

Single RE atoms on metal surfaces possess similar barriers for magnetization reversal as those on graphene/Ir(111). In particular, Er atoms on $\operatorname{Pt}(111)$ have $U_{\text {rev }}$ similar to the one of Dy atoms on graphene/ $\operatorname{Ir}(111), 5.3 \mathrm{meV}$ [10]. However, they do not show hysteresis in their magnetization curves at $2.5 \mathrm{~K}$, indicating a lifetime of order of seconds or less. This implies that the characteristic time $\tau_{0}$ for RE atoms on graphene is at least three orders of magnitude longer than the one on metal surfaces, possibly due to a stronger decoupling of the localized spins to both electrons and phonons of the surface. 


\section{CONCLUSIONS}

We combined XAS and multiplet calculations to study the interaction between RE atoms and graphene/Ir(111) and the resulting magnetic properties. Our study has shown the conditions that need to be fulfilled to achieve stable magnetization. Similar to what has been demonstrated for molecular magnets, $\mathrm{RE}$ atoms on graphene/ $\operatorname{Ir}(111)$ require a strong uniaxial anisotropy and a ground state protected from QTM. In addition, the system should have a suitable level scheme with a large $U_{\text {rev }}$ barrier in order to suppress thermally activated QTM. Finally, for the values of $U_{\text {rev }}$ of the investigated systems, the decoupling provided by graphene is crucial in reducing the scattering with electrons and phonons of the supporting metal substrate. Matching all these conditions is the key for identifying novel single atom magnets.

\section{ACKNOWLEDGMENTS}

We gratefully acknowledge funding by the Swiss National Science Foundation (SNSF) through Grants No. 200021_146715/1 (R.B.), No. 200020_157081/1 (A.S.), and No. PZ00P2_142474 (C.W. and J.D.). F.D. acknowledges funding support from the Institute for Basic Science under the project IBS-R027-D1.

\section{APPENDIX: CRYSTAL FIELD PARAMETERS}

In this Appendix, Tables II-VI provide the spatial coordinates and value of each charge used for the CF description of the different RE elements in the multiX code. Table VII provides the parameters describing the CF splitting in the Stevens operator representation.

TABLE II. Point charge CF scheme employed in multiplet calculations for Dy on graphene/ $\operatorname{Ir}(111)$ [19].

\begin{tabular}{cccc}
\hline \hline$x(\AA)$ & $y(\AA)$ & $z(\AA)$ & $q(e)$ \\
\hline 0 & 0 & -2 & 0.13 \\
1.4 & 0 & -2 & -0.22 \\
-1.4 & 0 & -2 & -0.22 \\
0.7 & 1.212436 & -2 & -0.22 \\
0.7 & -1.212436 & -2 & -0.22 \\
-0.7 & 1.212436 & -2 & -0.22 \\
-0.7 & -1.212436 & -2 & -0.22 \\
2.8 & 0 & -2.5 & 0.09 \\
-2.8 & 0 & -2.5 & 0.09 \\
1.4 & 2.424871 & -2.5 & 0.09 \\
1.4 & -2.424871 & -2.5 & 0.09 \\
-1.4 & 2.424871 & -2.5 & 0.09 \\
-1.4 & -2.424871 & -2.5 & 0.09 \\
2.8 & 2.424871 & -2.5 & -0.01 \\
2.8 & -2.424871 & -2.5 & -0.01 \\
3.5 & 1.212436 & -2.5 & -0.01 \\
3.5 & -1.212436 & -2.5 & -0.01 \\
0.7 & 3.637307 & -2.5 & -0.01 \\
0.7 & -3.637307 & -2.5 & -0.01 \\
-0.7 & 3.637307 & -2.5 & -0.01 \\
-0.7 & -3.637307 & -2.5 & -0.01 \\
-2.8 & 2.424871 & -2.5 & -0.01 \\
-2.8 & -2.424871 & -2.5 & -0.01 \\
-3.5 & 1.212436 & -2.5 & -0.01 \\
-3.5 & -1.212436 & -2.5 & -0.01 \\
\hline \hline
\end{tabular}

TABLE III. Point charge CF scheme employed in multiplet calculations for $\mathrm{Nd}$ on graphene/ $\operatorname{Ir}(111)$.

\begin{tabular}{cccc}
\hline \hline$x(\AA)$ & $y(\AA)$ & $z(\AA)$ & $q(e)$ \\
\hline 1.4 & 0 & -0.5 & -0.025 \\
-1.4 & 0 & -0.5 & -0.025 \\
0.7 & 1.212436 & -0.5 & -0.025 \\
0.7 & -1.212436 & -0.5 & -0.025 \\
-0.7 & 1.212436 & -0.5 & -0.025 \\
-0.7 & -1.212436 & -0.5 & -0.025 \\
\hline \hline
\end{tabular}

TABLE IV. Point charge CF scheme employed in multiplet calculations for Ho on graphene/Ir(111).

\begin{tabular}{cccc}
\hline \hline$x(\AA)$ & $y(\AA)$ & $z(\AA)$ & $q(e)$ \\
\hline 0 & 0 & -2 & -0.05 \\
1.4 & 0 & -2.5 & -0.12 \\
-1.4 & 0 & -2.5 & -0.12 \\
0.7 & 1.212436 & -2.5 & -0.12 \\
0.7 & -1.212436 & -2.5 & -0.12 \\
-0.7 & 1.212436 & -2.5 & -0.12 \\
-0.7 & -1.212436 & -2.5 & -0.12 \\
0 & 1.4 & -1.07 & 0.25 \\
0 & -1.4 & -1.07 & 0.25 \\
1.212436 & 0.7 & -1.07 & 0.25 \\
-1.212436 & 0.7 & -1.07 & 0.25 \\
1.212436 & -0.7 & -1.07 & 0.25 \\
-1.212436 & -0.7 & -1.07 & 0.25 \\
\hline \hline
\end{tabular}

TABLE V. Point charge CF scheme employed in multiplet calculations for Er on graphene/Ir(111).

\begin{tabular}{cccc}
\hline \hline$x(\AA)$ & $y(\AA)$ & $z(\AA)$ & $q(e)$ \\
\hline 0 & 0 & -1.3 & -0.0235 \\
1.4 & 0 & -2.0 & 0.01 \\
-1.4 & 0 & -2.0 & 0.01 \\
0.7 & 1.212436 & -2.0 & 0.01 \\
0.7 & -1.212436 & -2.0 & 0.01 \\
-0.7 & 1.212436 & -2.0 & 0.01 \\
-0.7 & -1.212436 & -2.0 & 0.01 \\
0 & 1.4 & -1.5 & -0.002 \\
0 & -1.4 & -1.5 & -0.002 \\
1.212436 & 0.7 & -1.5 & -0.002 \\
-1.212436 & 0.7 & -1.5 & -0.002 \\
1.212436 & -0.7 & -1.5 & -0.002 \\
-1.212436 & -0.7 & -1.5 & -0.002 \\
\hline \hline
\end{tabular}

TABLE VI. Point charge CF scheme employed in multiplet calculations for $\mathrm{Tb}$ on graphene/ $\operatorname{Ir}(111)$.

\begin{tabular}{cccc}
\hline \hline$x(\AA)$ & $y(\AA)$ & $z(\AA)$ & $q(e)$ \\
\hline 0 & 0 & -1.5 & -0.017 \\
1.4 & 0 & -1.5 & 0.034 \\
-1.4 & 0 & -1.5 & 0.034 \\
0.7 & 1.212436 & -1.5 & 0.034 \\
0.7 & -1.212436 & -1.5 & 0.034 \\
-0.7 & 1.212436 & -1.5 & 0.034 \\
-0.7 & -1.212436 & -1.5 & 0.034 \\
\hline
\end{tabular}


TABLE VII. Parameters describing the CF splitting in the Stevens operator representation for each investigated RE atom.

\begin{tabular}{ccccc}
\hline \hline Element & $B_{0}^{2}(\mu \mathrm{eV})$ & $B_{0}^{4}(\mathrm{neV})$ & $B_{0}^{6}(\mathrm{neV})$ & $B_{6}^{6}(\mathrm{neV})$ \\
\hline $\mathrm{Dy}$ & -121 & 100 & 1.5 & 0.3 \\
$\mathrm{Nd}$ & -359 & -90 & 60 & 1170 \\
$\mathrm{Ho}$ & 50 & 630 & -2.5 & 25 \\
$\mathrm{Er}$ & 152 & 260 & -2.7 & 0.2 \\
$\mathrm{~Tb}$ & 55 & 250 & 0.5 & $\leqslant 0.1$ \\
\hline \hline
\end{tabular}

[1] P. Gambardella, S. Rusponi, M. Veronese, S. S. Dhesi, C. Grazioli, A. Dallmeyer, I. Cabria, R. Zeller, P. H. Dederichs, K. Kern, C. Carbone, and H. Brune, Science 300, 1130 (2003).

[2] C. F. Hirjibehedin, C.-Y. Lin, A. F. Otte, M. Ternes, C. P. Lutz, B. A. Jones, and A. J. Heinrich, Science 317, 1199 (2007).

[3] F. Meier, L. Zhou, J. Wiebe, and R. Wiesendanger, Science 320, 82 (2008).

[4] A. A. Khajetoorians, S. Lounis, B. Chilian, A. T. Costa, L. Zhou, D. L. Mills, J. Wiebe, and R. Wiesendanger, Phys. Rev. Lett. 106, 037205 (2011).

[5] F. Donati, Q. Dubout, G. Autès, F. Patthey, F. Calleja, P. Gambardella, O. V. Yazyev, and H. Brune, Phys. Rev. Lett. 111, 236801 (2013).

[6] F. Donati, L. Gragnaniello, A. Cavallin, F. D. Natterer, Q. Dubout, M. Pivetta, F. Patthey, J. Dreiser, C. Piamonteze, S. Rusponi, and H. Brune, Phys. Rev. Lett. 113, 177201 (2014).

[7] I. G. Rau, S. Baumann, S. Rusponi, F. Donati, S. Stepanow, L. Gragnaniello, J. Dreiser, C. Piamonteze, F. Nolting, S. Gangopadhyay, O. R. Albertini, R. M. Macfarlane, C. P. Lutz, B. A. Jones, P. Gambardella, A. J. Heinrich, and H. Brune, Science 344, 988 (2014).

[8] G. E. Pacchioni, L. Gragnaniello, F. Donati, M. Pivetta, G. Autès, O. V. Yazyev, S. Rusponi, and H. Brune, Phys. Rev. B 91, 235426 (2015).

[9] T. Miyamachi, T. Schuh, T. Märkl, C. Bresch, T. Balashov, A. Stöhr, C. Karlewski, S. André, M. Marthaler, M. Hoffmann, M. Geilhufe, S. Ostanin, W. Hergert, I. Mertig, G. Schön, A. Ernst, and W. Wulfhekel, Nature 503, 242 (2013).

[10] F. Donati, A. Singha, S. Stepanow, C. Wäckerlin, J. Dreiser, P. Gambardella, S. Rusponi, and H. Brune, Phys. Rev. Lett. 113, 237201 (2014).

[11] M. Steinbrecher, A. Sonntag, M. dos Santos Dias, M. Bouhassoune, S. Lounis, J. Wiebe, R. Wiesendanger, and A. A. Khajetoorians, Nat. Commun. 7, 10454 (2015).

[12] N. Ishikawa, M. Sugita, T. Okubo, N. Tanaka, T. Ino, and Y. Kaizu, Inorg. Chem. 42, 2440 (2003).

[13] R. Westerström, J. Dreiser, C. Piamonteze, M. Muntwiler, S. Weyeneth, H. Brune, S. Rusponi, F. Nolting, A. Popov, S. F. Yang, L. Dunsch, and T. Greber, J. Am. Chem. Soc. 134, 9840 (2012).

[14] Y.-C. Chen, J.-L. Liu, L. Ungur, J. Liu, Q.-W. Li, L.-F. Wang, Z.-P. Ni, L. F. Chibotaru, X.-M. Chen, and M.-L. Tong, J. Am. Chem. Soc. 138, 2829 (2016).

[15] C. A. P. Goodwin, F. Ortu, D. Reta, N. F. Chilton, and D. P. Mills, Nature 548, 439 (2017).
[16] F.-S. Guo, B. M. Day, Y.-C. Chen, M.-L. Tong, A. Mansikkamäki, and R. A. Layfield, Angew. Chem. Int. Ed. 56, 11445 (2017).

[17] F. Donati, S. Rusponi, S. Stepanow, C. Wäckerlin, A. Singha, L. Persichetti, R. Baltic, K. Diller, F. Patthey, E. Fernandes, J. Dreiser, Ž. Šljivančanin, K. Kummer, C. Nistor, P. Gambardella, and H. Brune, Science 352, 318 (2016).

[18] F. D. Natterer, K. Yang, W. Paul, P. Willke, T. Choi, T. Greber, A. J. Heinrich, and C. P. Lutz, Nature 543, 226 (2017).

[19] R. Baltic, M. Pivetta, F. Donati, C. Wäckerlin, A. Singha, J. Dreiser, S. Rusponi, and H. Brune, Nano Lett. 16, 7610 (2016).

[20] A. L. Kozub, A. B. Shick, F. Máca, J. Kolorenč, and A. I. Lichtenstein, Phys. Rev. B 94, 125113 (2016).

[21] M. Karbowiak and C. Rudowicz, Phys. Rev. B 93, 184415 (2016).

[22] A. B. Shick, D. S. Shapiro, J. Kolorenc, and A. I. Lichtenstein, Sci. Rep. 7, 2751 (2017).

[23] A. B. Shick and A. I. Lichtenstein, J. Magn. Magn. Mater. 454, 61 (2018).

[24] X. Liu, C. Z. Wang, M. Hupalo, W. C. Lu, M. C. Tringides, Y. X. Yao, and K. M. Ho, Phys. Chem. Chem. Phys. 14, 9157 (2012).

[25] A. Abragam and B. Bleaney, Electron Paramagnetic Resonance of Transition Ions (Oxford University Press, Oxford, UK, 2012).

[26] M. Marciani, C. Hübner, and B. Baxevanis, Phys. Rev. B 95, 125433 (2017).

[27] D. Gatteschi and R. Sessoli, Angew. Chem. Int. Ed. 42, 268 (2003).

[28] L. Ungur and L. F. Chibotaru, Phys. Chem. Chem. Phys. 13, 20086 (2011).

[29] S. Rusponi, M. Papagno, P. Moras, S. Vlaic, M. Etzkorn, P. M. Sheverdyaeva, D. Pacilé, H. Brune, and C. Carbone, Phys. Rev. Lett. 105, 246803 (2010).

[30] A. Singha, Magnetic properties of surface-adsorbed single rare earth atoms, molecules, atomic scale clusters, Ph.D. thesis, EPFL, Switzerland, 2017.

[31] A. Singha, F. Donati, C. Wäckerlin, R. Baltic, J. Dreiser, M. Pivetta, S. Rusponi, and H. Brune, Nano Lett. 16, 3475 (2016).

[32] C. Piamonteze, U. Flechsig, S. Rusponi, J. Dreiser, J. Heidler, M. Schmidt, R. Wetter, M. Calvi, T. Schmidt, H. Pruchova, J. Krempasky, C. Quitmann, H. Brune, and F. Nolting, J. Synchrotron Rad. 19, 661 (2012).

[33] A. Uldry, F. Vernay, and B. Delley, Phys. Rev. B 85, 125133 (2012). 
[34] J. J. Baldoví, J. J. Borras-Almenár, J. M. Clemente-Juan, E. Coronado, and A. Gaita-Ariño, Dalton Trans. 41, 13705 (2012).

[35] N. F. Chilton, D. Collison, E. J. L. Mclnnes, R. E. P. Winpenny, and A. Soncini, Nat. Commun. 4, 2551 (2013).

[36] J. J. Baldoví, J. M. Clemente-Juan, E. Coronado, and A. GaitaAriño, Inorg. Chem. 53, 11323 (2014).

[37] N. Ishikawa, M. Sugita, T. Ishikawa, S.-y. Koshihara, and Y. Kaizu, J. Phys. Chem. B 108, 11265 (2005).

[38] R. Gaisch, J. K. Gimzewski, B. Reihl, R. R. Schlittler, M. Tschudy, and W. D. Schneider, Ultramicroscopy 42-44, 1621 (1992).

[39] A. Singha, R. Baltic, F. Donati, C. Wäckerlin, J. Dreiser, L. Persichetti, S. Stepanow, P. Gambardella, S. Rusponi, and H. Brune, Phys. Rev. B 96, 224418 (2017).

[40] H. Brune, Surf. Sci. Rep. 31, 125 (1998).

[41] M. Domke, C. Laubschat, M. Prietsch, T. Mandel, G. Kaindl, and W. D. Schneider, Phys. Rev. Lett. 56, 1287 (1986).

[42] J. Dreiser, R. Westerström, C. Piamonteze, F. Nolting, S. Rusponi, H. Brune, S. Yang, A. Popov, L. Dunsch, and T. Greber, Appl. Phys. Lett. 105, 032411 (2014).

[43] C. Wäckerlin, F. Donati, A. Singha, R. Baltic, S. Rusponi, K. Diller, F. Patthey, M. Pivetta, Y. Lan, S. Klyatskaya, M. Ruben, H. Brune, and J. Dreiser, Adv. Mater. 28, 5195 (2016).
[44] E. M. Chudnovsky, D. A. Garanin, and R. Schilling, Phys. Rev. B 72, 094426 (2005).

[45] B. T. Thole, G. van der Laan, J. C. Fuggle, G. A. Sawatzky, R. C. Karnatak, and J.-M. Esteva, Phys. Rev. B 32, 5107 (1985).

[46] J. B. Goedkoop, B. T. Thole, G. van der Laan, G. A. Sawatzky, F. M. F. de Groot, and J. C. Fuggle, Phys. Rev. B 37, 2086 (1988).

[47] A. Delin, L. Fast, B. Johansson, J. M. Wills, and O. Eriksson, Phys. Rev. Lett. 79, 4637 (1997).

[48] L. Peters, S. Ghosh, B. Sanyal, C. van Dijk, W. de Heer, A. Delin, I. Di Marco, O. Eriksson, M. I. Katsnelson, B. Johansson, and A. Kirilyuk, Sci. Rep. 6, 19676 (2016).

[49] S. Stepanow, J. Honolka, P. Gambardella, L. Vitali, N. Abdurakhmanova, T.-C. Tseng, S. Rauschenbach, S. L. Tait, V. Sessi, S. Klyatskaya, M. Ruben, and K. Kern, J. Am. Chem. Soc. 132, 11900 (2010).

[50] M. Mannini, F. Pineider, C. Danieli, F. Totti, L. Sorace, P. Sainctavit, M.-A. Arrio, E. Otero, L. Joly, J. C. Cezar, A. Cornia, and R. Sessoli, Nature 468, 417 (2010).

[51] C. Cervetti, A. Rettori, M. G. Pini, A. Cornia, A. Repollés, F. Luis, M. Dressel, S. Rauschenbach, K. Kern, M. Burghard, and L. Bogani, Nat. Mater. 15, 164 (2016). 